\title{
SDU
}

\section{Generic Finiteness of Equilibrium Distributions for Bimatrix Outcome Game Forms}

\author{
by \\ Cristian Litan, Francisco Marhuenda and Peter Sudhölter
}

Discussion Papers on Business and Economics

No. $7 / 2017$

FURTHER INFORMATION

Department of Business and Economics Faculty of Business and Social Sciences University of Southern Denmark Campusvej 55, DK-5230 Odense M

Denmark 


\title{
Generic Finiteness of Equilibrium Distributions for Bimatrix Outcome Game Forms*
}

\author{
Cristian Litan ${ }^{\dagger 1}$, Francisco Marhuenda ${ }^{\ddagger 2}$, and Peter Sudhölter ${ }^{\S 3}$ \\ ${ }^{1}$ Universitatea Babeş-Bolyai, Cluj-Napoca \\ ${ }^{2}$ University Carlos III of Madrid \\ ${ }^{3}$ University of Southern Denmark
}

May 16, 2017

\begin{abstract}
We provide sufficient and necessary conditions for the generic finiteness of the number of distributions on outcomes, induced by the completely mixed Nash equilibria associated to a bimatrix outcome game form. These equivalent conditions are stated in terms of the ranks of two matrices constructed from the original game form.
\end{abstract}

Keywords: Outcome game form, Completely mixed Nash equilibrium, Generic finiteness JEL Classification: C72

MSC2000 subject classification: 91A12

\section{Introduction}

It has long been established (Rosenmüller 1971, Wilson 1971, Harsanyi 1973) that for normal form games with an arbitrary number of players, if the payoffs can be independently perturbed, there is generically a finite number of equilibria. For the case of extensive form games Kreps and Wilson (1982) show that the equilibrium distributions on terminal nodes are generically finite.

The equivalent result for outcome games has turned out to be difficult to elucidate. On the one hand, Govindan and McLennan (2001) were the first to provide an example of a game form for which the Nash equilibria of the games associated to an open set of utility profiles induce a continuum of probability distributions on outcomes. Their example made use of three players and six outcomes. Examples of game forms with the same feature have appeared since: in Kukushkin, Litan, and Marhuenda (2008) with two players and four outcomes and in Litan, Marhuenda, and Sudhölter (2015) with three players and three outcomes.

\footnotetext{
* The first author acknowledges that this work was supported by a grant of the Romanian National Authority for Scientific Research and Innovation, CNCS-UEFISCDI, project number PN-II-RU-TE-2014-4-1827. The second author acknowledges financial support from the María de Maeztu grant MDM 2014-0431 and project ECO2013-42710-P, both from the Spanish Ministerio de Economía y Competitividad. The third author acknowledges support from the Spanish Ministerio de Economía y Competitividad under project ECO2015-66803-P and from the Danish Council for Independent Research-Social Sciences (Grant-id: DFF 1327-00097).

${ }^{\dagger}$ cristian.litan@econ.ubbcluj.ro

${ }_{\ddagger}^{\ddagger}$ marhuend@eco.uc3m.es

$\S \mathrm{psu} @$ sam.sdu.dk
} 
On the other hand, there are a number of results that point towards the paucity of such examples. Govindan and McLennan (2001) proved that for games with two outcomes and any number of players the number of equilibrium distributions on outcomes is generically finite. Similar results have been obtained for two player, three outcomes games (González-Pimienta 2010), sender-receiver cheap-talk games (Park 1997), zero sum or common interest games (Govindan and McLennan 1998, Litan and Marhuenda 2012) and games with three players and two strategies each (Litan, Marhuenda, and Sudhölter 2015).

Clarifying for what types of game forms the number of probability distributions on outcomes induced by the Nash equilibria of the associated game is generically finite remains an open problem. In the present work we address this question and provide a partial answer. We find sufficient and necessary conditions for the generic finiteness of the number of distributions on outcomes, induced by the completely mixed Nash equilibria associated to a bimatrix outcome game form. These are specified in terms of the ranks of two matrices constructed from the original game form and can be checked automatically.

\section{Outcome game forms with two players}

We follow very closely the notation and set up adopted in Litan, Marhuenda, and Sudhölter (2015) and Litan and Marhuenda (2012). Let $S^{1}=\{1,2, \ldots, m\}$ and $S^{2}=\{1,2, \ldots, n\}$ be the two players' sets of pure strategies. Let $S=S^{1} \times S^{2}$ and consider a finite set of outcomes $\Omega$. We denote by $\Delta(\Omega)$ (resp. $\Delta_{+}(\Omega)$ ) the set of (resp. strictly positive) probability measures on $\Omega$. An outcome game form is a function $\phi: S \rightarrow \Delta(\Omega)$. We write $\phi=\left(\phi^{\omega}\right)_{\omega \in \Omega}$ and for each outcome $\omega \in \Omega$ we regard $\phi^{\omega}$ as an $m \times n$ matrix, the entries of which are the probabilities that $\phi(i, j)(1 \leq i \leq n, 1 \leq j \leq m)$ assigns to the outcome $\omega \in \Omega$.

Agents have a utility function on outcomes $u \in \mathbb{R}^{\Omega}$, which extends to a von-Neumann-Morgenstern expected utility function. For each $u \in \mathbb{R}^{\Omega}$ we assign the matrix

$$
u(\phi)=\sum_{\omega \in \Omega} u(\omega) \phi^{\omega} .
$$

Given two profiles of utilities on outcomes $u^{1}, u^{2} \in \mathbb{R}^{\Omega}$ for the players, the matrices $u^{1}(\phi)$ and $u^{2}(\phi)$ define the two-person game $\left(u^{1}(\phi), u^{2}(\phi)\right)$. A pair of strategies $(x, y) \in \Delta\left(S^{1}\right) \times \Delta\left(S^{2}\right)$ is a Nash equilibrium (NE) of that game if $x u^{1}(\phi) y \geqslant u^{1}(\phi)_{i} . y$ and $x u^{2}(\phi) y \geqslant x u^{2}(\phi)_{\cdot j}$ for all $i \in S^{1}$ and $j \in S^{2}$. Here, $u(\phi)_{i}$. (resp. $u(\phi) \cdot j$ ) denote the $i$-th row (resp. $j$-th column) of the matrix $u(\phi)$. Throughout the paper, $x u(\phi)$ (resp. $u(\phi) y$ ) are regarded as elements of $\mathbb{R}^{n}$ (resp. $\mathbb{R}^{m}$ ) and the scalar product of $z, \bar{z} \in \mathbb{R}^{k}$ is written as $z \cdot \bar{z}$ or simply as $z \bar{z}$. The pair $(x, y)$ is a completely mixed $N E$ (CMNE) if, in addition, $x \in \Delta_{+}\left(S^{1}\right)$ and $y \in \Delta_{+}\left(S^{2}\right)$.

The strategies $x \in \Delta\left(S^{1}\right)$ and $y \in \Delta\left(S^{2}\right)$ of the players induce a probability distribution on $\Omega$ that assigns the probability $x \phi^{\omega} y$ to the outcome $\omega \in \Omega$.

We identify $\mathbb{R}^{\Omega}$ with Euclidean space $\mathbb{R}^{|\Omega|}$. With this identification the mapping $u \mapsto u(\phi)$ is linear from $\mathbb{R}^{|\Omega|}$ into the linear space of $m \times n$ matrices. We say that a subset of $\mathbb{R}^{\Omega}$ is generic if it contains an open and dense subset. For $l \in \mathbb{N}$, let $d_{l}$ denote the vector $(1, \ldots, 1) \in \mathbb{R}^{l}$.

The number of pure NEs of a finite game is finite. In the case of games with a mixed NE, by eliminating those strategies that are played with zero probability, we will focus on the CMNEs of the corresponding subgames. Given two utility profiles $u^{1}, u^{2} \in \mathbb{R}^{\Omega}$, if a pair $(x, y) \in \Delta_{+}\left(S^{1}\right) \times \Delta_{+}\left(S^{2}\right)$ of completely mixed strategies is a NE of the game $\left(u^{1}(\phi), u^{2}(\phi)\right)$, then it is a solution of the following systems of linear 
equations.

$$
\begin{array}{ll}
u^{1}(\phi) y=\alpha_{1} d_{m}, & y \cdot d_{n}=1 \\
x u^{2}(\phi)=\alpha_{2} d_{n}, & x \cdot d_{m}=1
\end{array}
$$

for some $\alpha_{i} \in \mathbb{R}$, the payoff of player $i=1,2$.

Definition 2.1. Given an outcome game form $\phi$ and a pair of utility profiles $u^{1}$, $u^{2} \in \mathbb{R}^{\Omega}$, a $q u a s i$ equilibrium (QE) of the game $\left(u^{1}(\phi), u^{2}(\phi)\right)$ is a pair of solutions $(x, y)=\left(x\left(u^{2}\right), y\left(u^{1}\right)\right) \in \mathbb{R}^{m} \times \mathbb{R}^{n}$ of the system of equations (1) and (2), for some $\alpha_{i}=\alpha_{i}\left(u^{i}\right) \in \mathbb{R}, i=1,2$.

We say that vector $z \in \mathbb{R}^{\Omega}$ is a quasi(probability)-distribution on $\Omega$ for the game $\left(u^{1}(\phi), u^{2}(\phi)\right)$ if there exists a $\mathrm{QE}(x, y)$ of that game such that for each $\omega \in \Omega, z(\omega)=x \phi^{\omega} y$.

Thus, the set of CMNE is a subset of the set of all QE of a given game. For the rest of the paper we fix an outcome game form $\phi$. Given an $m \times n$ matrix $A$ and $b \in \mathbb{R}^{m},(A \mid b)$ denotes the $m \times(n+1)$ matrix that arises from $A$ by adding $b$ as final column and $A^{t}$ denotes the transpose matrix of $A$. Let $k=\max \left\{\operatorname{rank} u(\phi): u \in \mathbb{R}^{\Omega}\right\}$. We rely on the following fact shown by Mas-Colell (2010). There is an open and dense (and hence generic) subset $G$ of $\mathbb{R}^{\Omega}$ such that the following conditions hold.

(a) For every $u \in G$, we have $\operatorname{rank} u(\phi)=k$. After reordering, if necessary, the strategies of the players we may write

$$
u(\phi)=\left(\begin{array}{ll}
B & C \\
D & E
\end{array}\right)
$$

where $B=B(u)$ is a $k \times k$ matrix with $|B| \neq 0 .^{1}$

(b) The functions $k_{1}=\operatorname{rank}\left(u(\phi) \mid d_{m}\right)$ and $k_{2}=\operatorname{rank}\left(u(\phi)^{t} \mid d_{n}\right)$ are constant on $G$.

Throughout the rest of this exposition, we fix a reordering of the strategies of the players such that (a) holds. Consider the following polynomial on $|\Omega|$ variables,

$$
p(u)=|B(u)| d_{k} B^{-1}(u) d_{k}, \quad u \in \mathbb{R}^{\Omega}
$$

Whenever $p(u) \neq 0$ we define

$$
\alpha(u)=\frac{|B(u)|}{p(u)}
$$

We have the following.

Proposition 2.2. If $k=m=n$, then generically there is at most one $Q E$ and, hence, at most one CMNE. Suppose $k<\max \{m, n\}$. Then,

(a) If $k<\max \left\{k_{1}, k_{2}\right\}$, then for every $u^{1}, u^{2} \in G$ the game $\left(u^{1}(\phi), u^{2}(\phi)\right)$ has no QE and, hence, no CMNE.

(b) If $k_{1}=k_{2}=k$ and $p$ is the zero polynomial, then for any $u^{1}, u^{2} \in G$ the game $\left(u^{1}(\phi), u^{2}(\phi)\right)$ has no $\mathrm{QE}$ and, hence, no CMNE.

\footnotetext{
${ }^{1}$ When there is no danger of confusion, we will not write explicitly the dependence on the utility $u$ for matrices.
} 
(c) Let $u^{1}, u^{2} \in G$. Suppose $k_{1}=k_{2}=k$ and for $i=1,2, p\left(u^{i}\right) \neq 0$. Then, there is a continuum of $\mathrm{QE}$ s of the game $\left(u^{1}(\phi), u^{2}(\phi)\right)$. Furthermore, the systems of linear equations (1) and (2) have a solution only if the payoffs are $\alpha_{i}=\alpha\left(u^{i}\right), i=1,2$, with $\alpha$ defined in (5).

Proof. The case $k_{1}=k_{2}=k$ of the Proposition is a standard result in elementary Linear Algebra. See Lemmas 3.2, 3.3 and 3.4 in Litan and Marhuenda (2012) for the proof of parts (b) and (c).

We prove next part (a). We consider only the case $k_{1}=k+1$. The case $k_{2}=k+1$ is similar. We remark first that, since $d_{m}$ is not a linear combination of the columns of $u(\phi)$, any $y \in \mathbb{R}^{n}$ which is a solution of (1) for some $u \in G$, must satisfy $u(\phi) y=0$.

Assume now that there are $u^{1}, u^{2} \in G$ such that the game $\left(u^{1}(\phi), u^{2}(\phi)\right)$ has a QE $(x, y)$ with $x \in \mathbb{R}^{m}$ and $y \in \mathbb{R}^{n}$. Then, $u^{1}(\phi) y=0$ by the previous remark. Since, $G$ is open, there exists $\varepsilon>0$ such that $\bar{u}=u^{1}+\varepsilon d_{|\Omega|} \in G$. Moreover, $(x, y)$ is also a $\mathrm{QE}$ of the game $\left(\bar{u}(\phi), u^{2}(\phi)\right)$ and $\bar{u}(\phi) y=\varepsilon d_{m}$. But, this contradicts the remark in the previous paragraph. Hence, part (a) of the Proposition follows.

Since, $k_{1}, k_{2} \in\{k, k+1\}$ and we are only interested in the existence of a continuum of CMNEs, from now we consider only games for wich the following holds.

\section{Assumption 2.3.}

- $k_{1}=k_{2}=k<\max \{m, n\}$.

- The polynomial $p$ in (4) is not the zero polynomial.

Note that, under the above assumption, the set $U=\{u \in G: p(u) \neq 0\}$ is open and dense in $\mathbb{R}^{|\Omega|}$. Hence, it is generic. Let $\alpha: U \rightarrow \mathbb{R}$ as in (5). Define the functions $y^{p}: U \rightarrow \mathbb{R}^{n}, y^{h}: U \times \mathbb{R}^{n-k} \rightarrow \mathbb{R}^{n}$, $x^{p}: U \rightarrow \mathbb{R}^{m}$ and $x^{h}: U \times \mathbb{R}^{m-k} \rightarrow \mathbb{R}^{m}$ by

$$
\begin{aligned}
y^{p}(u) & =\left(\alpha(u) B^{-1}(u) d_{k}, 0\right) \\
y^{h}(u, v) & =\left(-B^{-1}(u) C(u) v, v\right) \\
x^{p}(u) & =\left(\alpha(u) d_{k} B^{-1}(u), 0\right) \\
x^{h}(u, w) & =\left(-w D(u) B^{-1}(u), w\right)
\end{aligned}
$$

The following is proved in Lemmas 3.3 and 3.4 of Litan and Marhuenda (2012).

Lemma 2.4. Let Assumption 2.3 hold and let $u \in U$. Then,

(a) $u(\phi) y^{p}(u)=\alpha(u) d_{m}$ and $d_{n} \cdot y^{p}(u)=1$;

(b) $x^{p}(u) u(\phi)=\alpha(u) d_{n}$ and $d_{m} \cdot x^{p}(u)=1$;

(c) For every $v \in \mathbb{R}^{n-k}$ we have $u(\phi) y^{h}(u, v)=0$ and $d_{n} \cdot y^{h}(u, v)=0$;

(d) For every $w \in \mathbb{R}^{m-k}$ we have $x^{h}(u, w) u(\phi)=0$ and $d_{m} \cdot x^{h}(u, w)=0$;

For $V \subset \mathbb{R}^{l}$ a linear subspace and $a \in \mathbb{R}^{l}$ we let $a V=V a=\{a \cdot v: v \in V\}$. From now on, we follow the notation of Lemma 2.4. Given $u \in U$ we define $K_{1}(u)=\left\{z \in \mathbb{R}^{n}: u(\phi) z=0\right\}$ and $K_{2}(u)=\left\{t \in \mathbb{R}^{m}\right.$ : $t u(\phi)=0\}$. Since, we are assuming that $k_{1}=k_{2}=k$, we have that $K_{1}(u)=\operatorname{ker} u(\phi)=\operatorname{ker}\left(u(\phi) \mid d_{m}\right)$ 
and $K_{2}(u)=\operatorname{ker} u(\phi)^{t}=\operatorname{ker}\left(u(\phi)^{t} \mid d_{n}\right)$. We see from Lemma 2.4 that $K_{1}(u)=\left\{y^{h}(u, v): v \in \mathbb{R}^{n-k}\right\}$ and $K_{2}(u)=\left\{x^{h}(u, w): w \in \mathbb{R}^{m-k}\right\}$. It follows that $\operatorname{dim} K_{1}(u)=n-k, \operatorname{dim} K_{2}(u)=m-k$. and $K_{1}(u) d_{n}=d_{m} K_{2}(u)=0$. Let $u^{1}, u^{2} \in U$. Any $\mathrm{QE}(x, y)$ of the game $\left(u^{1}(\phi), u^{2}(\phi)\right)$ may be written as

$$
\left(x^{p}\left(u^{2}\right)+x^{h}\left(u^{2}, w\right), y^{p}\left(u^{1}\right)+y^{h}\left(u^{1}, v\right)\right)
$$

for some $v \in \mathbb{R}^{n-k}$ and $w \in \mathbb{R}^{m-k}$. When there is no danger of confusion we will not write explicitly the dependence on $u^{1}, u^{2}, v$ or $w$. For each outcome $\omega \in \Omega$, define the following polynomial in the variables $(v, w) \in \mathbb{R}^{n-k} \times \mathbb{R}^{m-k}$

$$
q_{\omega}(v, w)=x^{p}\left(u^{2}\right) \phi^{\omega} y^{p}\left(u^{1}\right)+x^{p}\left(u^{2}\right) \phi^{\omega} y^{h}\left(u^{1}, v\right)+x^{h}\left(u^{2}, w\right) \phi^{\omega} y^{p}\left(u^{1}\right)+x^{h}\left(u^{2}, w\right) \phi^{\omega} y^{h}\left(u^{1}, v\right)
$$

The probability that outcome $\omega \in \Omega$ occurs is $q_{\omega}(v, w)$. Given $x \in \mathbb{R}^{m}, y \in \mathbb{R}^{n}$ we define the following subspaces.

$$
\begin{aligned}
x \phi^{\omega} K_{1}\left(u^{1}\right) & =\left\{x \phi^{\omega} z: z \in K_{1}\left(u^{1}\right)\right\} \\
K_{2}\left(u^{2}\right) \phi^{\omega} y & =\left\{t \phi^{\omega} y: t \in K_{2}\left(u^{2}\right)\right\} \\
K_{2}\left(u^{2}\right) \phi^{\omega} K_{1}\left(u^{1}\right) & =\left\{t \phi^{\omega} z: z \in K_{1}\left(u^{1}\right), t \in K_{2}\left(u^{2}\right)\right\}
\end{aligned}
$$

Lemma 2.5. Suppose that Assumption 2.3 holds. Let $\omega \in \Omega, u^{1}, u^{2} \in U$. If either of the following two conditions hold

(a) $x\left(u^{2}\right) \phi^{\omega} K_{1}\left(u^{1}\right)=\{0\}$ for every solution $x\left(u^{2}\right)$ of the system of equations (2).

(b) $K_{2}\left(u^{2}\right) \phi^{\omega} y\left(u^{1}\right)=\{0\}$ for every solution $y\left(u^{1}\right)$ of the system of equations (1).

then, $K_{2}\left(u^{2}\right) \phi^{\omega} K_{1}\left(u^{1}\right)=\{0\}$.

Proof. Suppose that condition (a) holds. Let $t \in K_{2}\left(u^{2}\right)$. Recall that we may write $t$ as $t=x_{1}-x_{2}$, where $x_{1}, x_{2}$ are two solutions of the system of equations (2). Let $z \in K_{1}\left(u^{1}\right)$. Then, $t \phi^{\omega} z=x_{1} \phi^{\omega} z-x_{2} \phi^{\omega} z=$ $\alpha d_{n} z-\alpha d_{n} z=0$. Hence, it follows that $K_{2}\left(u^{2}\right) \phi^{\omega} K_{1}\left(u^{1}\right)=\{0\}$. Similarly, condition (b) implies that $K_{2}\left(u^{2}\right) \phi^{\omega} K_{1}\left(u^{1}\right)=\{0\}$.

Proposition 2.6. Suppose that Assumption 2.3 holds. Let $u^{1}, u^{2} \in U$. The set of $Q E$ of the game defined by $u^{1}(\phi)$ and $u^{2}(\phi)$ induce finitely many quasi-distributions on $\Omega$ if and only if the following two conditions hold.

(a) $x\left(u^{2}\right) \phi^{\omega} K_{1}\left(u^{1}\right)=\{0\}$ for every $\omega \in \Omega$ and every solution $x\left(u^{2}\right)$ of the system of equations (2).

(b) $K_{2}\left(u^{2}\right) \phi^{\omega} y\left(u^{1}\right)=\{0\}$ for every $\omega \in \Omega$ and for every solution $y\left(u^{1}\right)$ of the system of equations (1).

Proof. Since, the set of QE's of the game $\left(u^{1}(\phi), u^{2}(\phi)\right)$ is convex we have that the set

$$
\left\{z \in \mathbb{R}^{\Omega}: z \text { is a quasi-distribution on } \Omega \text { for the game }\left(u^{1}(\phi), u^{2}(\phi)\right)\right\}
$$

is connected. Hence, the QEs induce finitely many quasi-distributions on outcomes if and only if they induce a unique quasi-distribution on outcomes. We prove next part (a). Assume there is a unique quasi-distribution induced on outcomes by the QEs of the game. Let $x=x\left(u^{2}\right)$ be a solution of $(2)$, and 
$z=z\left(u^{1}\right) \in K_{1}\left(u^{1}\right)$. We write $z=y_{1}-y_{2}$, where $y_{1}, y_{2}$ are two solutions of (1). Then, for each $\omega \in \Omega$, we have that $x \phi^{\omega} y_{1}=x \phi^{\omega} y_{2}$. So,

$$
x \phi^{\omega} z=x \phi^{\omega} y_{1}-x \phi^{\omega} y_{2}=0
$$

Thus, $x \phi^{\omega} K_{1}\left(u^{1}\right)=\{0\}$ and (a) follows. Similarly, we can prove (b).

Conversely, suppose that conditions (a) and (b) hold. Let $u^{1}, u^{2} \in U$ and $\omega \in \Omega$. By Lemma 2.5 we have that $K_{2}\left(u^{2}\right) \phi^{\omega} K_{1}\left(u^{1}\right)=\{0\}$. Therefore, in (7), for every QE $\left(x^{p}\left(u^{2}\right)+x^{h}\left(u^{2}, w\right), y^{p}\left(u^{1}\right)+y^{h}\left(u^{1}, v\right)\right)$, we have that

$$
\left(x^{p}\left(u^{2}\right)+x^{h}\left(u^{2}, w\right)\right) \phi^{\omega}\left(y^{p}\left(u^{1}\right)+y^{h}\left(u^{1}, v\right)\right)=x^{p}\left(u^{2}\right) \phi^{\omega} y^{p}\left(u^{1}\right)
$$

And the QEs of the game defined by $u^{1}(\phi)$ and $u^{2}(\phi)$ induce a unique quasi-distribution on $\Omega$.

Corollary 2.7. Let Assumption 2.3 hold. Suppose there are two sets of vectors $V_{1} \subset \mathbb{R}^{n}$ and $V_{2} \subset \mathbb{R}^{m}$ such that for every $u \in U$ and $i=1,2$ we have that $V_{i}$ generates $K_{i}(u)$. Then, for any $u^{1}, u^{2} \in U$ the set of CMNE of the game $\left(u^{1}(\phi), u^{2}(\phi)\right)$ induce finitely many probability distributions on outcomes.

Proof. Let $z \in V_{1}$ and $t \in V_{2}$. For every $u \in U$, we have that $t u(\phi)=u(\phi) z=0$. Since, the entries of $u(\phi)$ are linear in $u$ and $U$ contains an open subset of $\mathbb{R}^{|\Omega|}$, we see that $t \phi^{\omega}=\phi^{\omega} z=0$ for every $\omega \in \Omega$. Hence, $\phi^{\omega} K_{1}(u)=K_{2}(u) \phi^{\omega}=\{0\}$ for every $u \in U$ and $\omega \in \Omega$. The result follows now from Proposition 2.6.

The following result provides necessary and sufficient conditions for the existence of finitely many probability distributions on outcomes in games induced by outcome game forms.

Theorem 2.8. Let Assumption 2.3 hold, $u^{1}, u^{2} \in U$ and suppose there is, at least, one CMNE of the game $\left(u^{1}(\phi), u^{2}(\phi)\right)$. Then, the set of all the CMNE of that game induces finitely many probability distributions on outcomes iff for every $\omega \in \Omega$

$$
\operatorname{rank}\left(\begin{array}{cc}
u^{1}(\phi) & 0 \\
\phi^{\omega} & u^{2}(\phi) \\
0 & d_{n}
\end{array}\right)=2 k \quad \text { and } \quad \operatorname{rank}\left(\begin{array}{ccc}
u^{2}(\phi) & \phi^{\omega} & 0 \\
0 & u^{1}(\phi) & d_{m}
\end{array}\right)=2 k
$$

The proof is based on techniques developed in Litan and Marhuenda (2012). A review of these and the details of the proof are provided in the appendix.

\section{References}

GonzÁlez-Pimienta, C. (2010): "Generic finiteness of outcome distributions for two person game forms with three outcomes", Mathematical Social Sciences, 59, 364-365.

Govindan, S., And A. McLennan (1998): "Generic finiteness of outcome distributions for two person game forms with zero sum and common interest utilities", Mimeo, University of Western Ontario.

(2001): "On the generic finiteness of equilibrium outcome distributions in game forms", Econometrica, 69, 455-471. 
HARSANYI, J. C. (1973): "Oddness of the number of equilibrium points: a new proof", International Journal of Game Theory, 2, 235-250.

Kreps, D. M., and R. WiLson (1982): "Sequential equilibria", Econometrica, 50, 863-894.

Kukushinin, N. S., C. Litan, and F. Marhuenda (2008): "On the generic finiteness of equilibrium outcome distributions in bimatrix game forms", Journal of Economic Theory, 139, 392-395.

Litan, C., F. Marhuenda, and P. Sudhölter (2015): "Determinacy of equilibrium in outcome game forms", Journal of Mathematical Economics, 60, 28-32.

Litan, C. M., And F. Marhuenda (2012): "Determinacy of equilibrium outcome distributions for zero sum and common utility games", Economics Letters, 115, 152 - 154.

Mas-Colell, A. (2010): "Generic finiteness of equilibrium payoffs for bimatrix games", Journal of Mathematical Economics, 46, 382-383.

PARK, I.-U. (1997): "Generic finiteness of equilibrium outcome distributions for sender-receiver cheaptalk games", Journal of Economic Theory, 76, 431-448.

Rosenmüller, J. (1971): "On a generalization of the Lemke-Howson algorithm to noncooperative nperson games", SIAM Journal on Applied Mathematics, 20, 73 - 79.

Wilson, R. (1971): "Computing equilibria of n-person games", SIAM Journal on Applied Mathematics, $20,80-87$.

\section{A The proof of Theorem 2.8}

Lemma A.1. Suppose that Assumption 2.3 holds. Let $u^{1}, u^{2} \in U$. The set of QE of the game $\left(u^{1}(\phi), u^{2}(\phi)\right)$ induce finitely many quasi-distributions on outcomes if and only if for every $\omega \in \Omega$ and every $Q E\left(x\left(u^{2}\right), y\left(u^{1}\right)\right)$ of that game the following two conditions hold.

(a) $x\left(u^{2}\right) \phi^{\omega}$ is in the image of $u^{1}(\phi)^{t}$

(b) $\phi^{\omega} y\left(u^{1}\right)$ is in the image of $u^{2}(\phi)$.

Proof. Let $u^{1}, u^{2} \in U$. By Proposition 2.6, the set of QE of the game $\left(u^{1}(\phi), u^{2}(\phi)\right)$ induce finitely many quasi-distributions on outcomes if and only if for every $\omega \in \Omega$, every solution $x\left(u^{2}\right)$ of the system of equations (2) and every solution $y\left(u^{1}\right)$ of the system of equations (1) we have that $x\left(u^{2}\right) \phi^{\omega} K_{1}\left(u^{1}\right)=\{0\}$ and $K_{2}\left(u^{2}\right) \phi^{\omega} y\left(u^{1}\right)=\{0\}$. This is equivalent to the statement that $x\left(u^{2}\right) \phi^{\omega}$ is orthogonal to $K_{1}\left(u^{1}\right)$ and $\phi^{\omega} y\left(u^{1}\right)$ is orthogonal to $K_{2}\left(u^{2}\right)$, which occurs if and only if $x\left(u^{2}\right) \phi^{\omega}$ is in the image of $u^{1}(\phi)^{t}$ and $\phi^{\omega} y\left(u^{1}\right)$ is in the image of $u^{2}(\phi)$.

Lemma A.2. Let $\omega \in \Omega$ and $u^{1}, u^{2} \in U$. Let Assumption 2.3 hold and suppose that $K_{2}\left(u^{2}\right) \phi^{\omega} K_{1}\left(u^{1}\right)=$ $\{0\}$. Then,

$$
\operatorname{rank}\left(\begin{array}{cc}
u^{1}(\phi) & 0 \\
\phi^{\omega} & u^{2}(\phi)
\end{array}\right)=2 k
$$


Proof. We use the notation

$$
\phi^{\omega}=\left(\begin{array}{ll}
B^{\omega} & C^{\omega} \\
D^{\omega} & E^{\omega}
\end{array}\right)
$$

to denote the decomposition of the matrix $u(\phi)$ in (3) applied to the matrix $\phi^{\omega}$. Let

$$
F=\left(\begin{array}{cc}
u^{1}(\phi) & 0 \\
\phi^{\omega} & u^{2}(\phi)
\end{array}\right)
$$

We can write now

$$
F=\left(\begin{array}{cccc}
B\left(u^{1}\right) & C\left(u^{1}\right) & 0 & 0 \\
D\left(u^{1}\right) & E\left(u^{1}\right) & 0 & 0 \\
B^{\omega} & C^{\omega} & B\left(u^{2}\right) & C\left(u^{2}\right) \\
D^{\omega} & E^{\omega} & D\left(u^{2}\right) & E\left(u^{2}\right)
\end{array}\right)
$$

By elementary row and column operations,

$$
\operatorname{rank} F=\operatorname{rank}\left(\begin{array}{ccc}
B\left(u^{1}\right) & C\left(u^{1}\right) & 0 \\
B^{\omega} & C^{\omega} & B\left(u^{2}\right) \\
D^{\omega} & E^{\omega} & D\left(u^{2}\right)
\end{array}\right)=\operatorname{rank}\left(\begin{array}{ccc}
B\left(u^{1}\right) & C\left(u^{1}\right) & 0 \\
B^{\omega} & C^{\omega} & B\left(u^{2}\right) \\
D_{1}^{\omega} & E_{1}^{\omega} & 0
\end{array}\right)
$$

where

$$
\begin{aligned}
& D_{1}^{\omega}=D^{\omega}-D\left(u^{2}\right) B^{-1}\left(u^{2}\right) B^{\omega} \\
& E_{1}^{\omega}=E^{\omega}-D\left(u^{2}\right) B^{-1}\left(u^{2}\right) C^{\omega}
\end{aligned}
$$

Finally,

$$
\operatorname{rank} F=\operatorname{rank}\left(\begin{array}{ccc}
B\left(u^{1}\right) & 0 & 0 \\
B^{\omega} & C_{2}^{\omega} & B\left(u^{2}\right) \\
D_{1}^{\omega} & E_{2}^{\omega} & 0
\end{array}\right)
$$

with $C_{2}^{\omega}=C^{\omega}-B^{\omega} B^{-1}\left(u^{1}\right) C\left(u^{1}\right)$ and

$$
\begin{aligned}
E_{2}^{\omega} & =E_{1}^{\omega}-D_{1}^{\omega} B^{-1}\left(u^{1}\right) C\left(u^{1}\right) \\
& =E^{\omega}-D\left(u^{2}\right) B^{-1}\left(u^{2}\right) C^{\omega}-D^{\omega} B^{-1}\left(u^{1}\right) C\left(u^{1}\right)+D\left(u^{2}\right) B^{-1}\left(u^{2}\right) B^{\omega} B^{-1}\left(u^{1}\right) C\left(u^{1}\right) \\
& =\left(\begin{array}{ll}
-D\left(u^{2}\right) B^{-1}\left(u^{2}\right) & I_{m-k}
\end{array}\right)\left(\begin{array}{cc}
B^{\omega} & C^{\omega} \\
D^{\omega} & E^{\omega}
\end{array}\right)\left(\begin{array}{c}
-B^{-1}\left(u^{1}\right) C\left(u^{1}\right) \\
I_{n-k}
\end{array}\right) \\
& =\left(\begin{array}{ll}
-D\left(u^{2}\right) B^{-1}\left(u^{2}\right) & I_{m-k}
\end{array}\right) \phi^{\omega}\left(\begin{array}{c}
-B^{-1}\left(u^{1}\right) C\left(u^{1}\right) \\
I_{n-k}
\end{array}\right)
\end{aligned}
$$

It follows from Lemma 2.4 that

$$
K_{1}\left(u^{1}\right)=\left\{y^{h}\left(u^{1}, v\right): v \in \mathbb{R}^{n-k}\right\}=\left\{\left(\begin{array}{c}
-B^{-1}\left(u^{1}\right) C\left(u^{1}\right) \\
I_{n-k}
\end{array}\right) v: v \in \mathbb{R}^{n-k}\right\}
$$

and

$$
K_{2}\left(u^{2}\right)=\left\{x^{h}\left(u^{2}, w\right): w \in \mathbb{R}^{m-k}\right\}=\left\{w\left(-D\left(u^{2}\right) B^{-1}\left(u^{2}\right) \quad I_{m-k}\right): w \in \mathbb{R}^{m-k}\right\}
$$


Since $K_{2}\left(u^{2}\right) \phi^{\omega} K_{1}\left(u^{1}\right)=\{0\}$, for any $v \in \mathbb{R}^{n-k}$ and $w \in \mathbb{R}^{m-k}$ we have that

$$
w E_{2}^{\omega} v=w\left(\begin{array}{ll}
-D\left(u^{2}\right) B^{-1}\left(u^{2}\right) & I_{m-k}
\end{array}\right) \phi^{\omega}\left(\begin{array}{c}
-B^{-1}\left(u^{1}\right) C\left(u^{1}\right) \\
I_{n-k}
\end{array}\right) v=0
$$

Therefore, $E_{2}^{\omega}=0$ and

$$
\operatorname{rank} F=\operatorname{rank}\left(\begin{array}{ccc}
B\left(u^{1}\right) & 0 & 0 \\
B^{\omega} & C_{2}^{\omega} & B\left(u^{2}\right) \\
D_{1}^{\omega} & 0 & 0
\end{array}\right)=\operatorname{rank}\left(\begin{array}{ccc}
B\left(u^{1}\right) & 0 & 0 \\
B^{\omega} & C_{2}^{\omega} & B\left(u^{2}\right)
\end{array}\right)=2 k
$$

because, since $\operatorname{rank} B\left(u^{1}\right)=\operatorname{rank} B\left(u^{2}\right)=k$, the rows of $D_{1}^{\omega}$ are a linear combination of the rows of $B\left(u^{1}\right)$.

Lemma A.3. Suppose that Assumption 2.3 holds. Let $u^{1}, u^{2} \in U, \omega \in \Omega$. Then,

(a) $x\left(u^{2}\right) \phi^{\omega} K_{1}\left(u^{1}\right)=\{0\}$ for every solution $x\left(u^{2}\right)$ of the system of equations (2) if and only if

$$
\operatorname{rank}\left(\begin{array}{cc}
u^{1}(\phi) & 0 \\
\phi^{\omega} & u^{2}(\phi) \\
0 & d_{n}
\end{array}\right)=2 k
$$

(b) $K_{2}\left(u^{2}\right) \phi^{\omega} y\left(u^{1}\right)=0$ for every solution $y\left(u^{1}\right)$ of the system of equations (1) if and only if

$$
\operatorname{rank}\left(\begin{array}{ccc}
u^{2}(\phi) & \phi^{\omega} & 0 \\
0 & u^{1}(\phi) & d_{m}
\end{array}\right)=2 k
$$

Proof. We prove only part (a). The proof of part (b) is similar. Fix a solution $x=x\left(u^{2}\right)$ of the system of equations (2). Let

$$
F=\left(\begin{array}{cc}
u^{1}(\phi) & 0 \\
\phi^{\omega} & u^{2}(\phi) \\
0 & d_{n}
\end{array}\right)
$$

Since, $d_{n}=\frac{1}{\alpha\left(u^{2}\right)} x u^{2}(\phi)$, by elementary row operations we have that

$$
\operatorname{rank} F=\operatorname{rank}\left(\begin{array}{cc}
u^{1}(\phi) & 0 \\
\phi^{\omega} & u^{2}(\phi) \\
\frac{1}{\alpha\left(u^{2}\right)} x \phi^{\omega} & 0
\end{array}\right)
$$

Assume that $x \phi^{\omega} K_{1}\left(u^{1}\right)=0$. Then, by Lemma A.1, $x \phi^{\omega}$ is in the image of $u^{1}(\phi)^{t}$ and hence $x \phi^{\omega}$ is a linear combination of the rows of $u^{1}(\phi)$. Therefore,

$$
\operatorname{rank} F=\operatorname{rank}\left(\begin{array}{cc}
u^{1}(\phi) & 0 \\
\phi^{\omega} & u^{2}(\phi)
\end{array}\right)
$$

and, by Lemmas 2.5 and A.2 we have that $\operatorname{rank} F=2 k$. 
Conversely, suppose now that $\operatorname{rank} F=2 k$. We proceed now as in Lemma A.2 and write $F$ as

$$
F=\left(\begin{array}{cccc}
u^{1}(\phi) & 0 & 0 \\
B^{\omega} & C^{\omega} & B\left(u^{2}\right) & C\left(u^{2}\right) \\
D^{\omega} & E^{\omega} & D\left(u^{2}\right) & E\left(u^{2}\right) \\
0 & 0 & d_{k} & d_{n-k}
\end{array}\right)
$$

The above argument shows that

$$
\operatorname{rank} F=\operatorname{rank}\left(\begin{array}{cccc}
u^{1}(\phi) & 0 & 0 \\
B^{\omega} & C^{\omega} & B\left(u^{2}\right) & C\left(u^{2}\right) \\
D^{\omega} & E^{\omega} & D\left(u^{2}\right) & E\left(u^{2}\right) \\
\frac{1}{\alpha\left(u^{2}\right)} x\left(u^{2}\right) \phi^{\omega} & 0 & 0
\end{array}\right)
$$

Since $\operatorname{rank} u^{2}(\phi)=\operatorname{rank} B\left(u^{2}\right)=k$, we get that

$$
\operatorname{rank} F=\operatorname{rank}\left(\begin{array}{ccc}
u^{1}(\phi) & 0 \\
B_{1}^{\omega} & C_{1}^{\omega} & B\left(u^{2}\right) \\
D_{1}^{\omega} & E_{1}^{\omega} & 0 \\
\frac{1}{\alpha\left(u^{2}\right)} x \phi^{\omega} & 0
\end{array}\right)=2 k
$$

where $D_{1}^{\omega}, E_{1}^{\omega}$ are defined in (8) and (9). Since, $\operatorname{rank} u^{1}(\phi)=\operatorname{rank} B\left(u^{2}\right)=k$, the rows of the matrix $\left(\begin{array}{cc}D_{1}^{\omega} & E_{1}^{\omega}\end{array}\right)$ and $x \phi^{\omega}$ are a linear combination of the rows of $u^{1}(\phi)$. It follows that $x \phi^{\omega}$ is orthogonal to $K_{1}\left(u^{1}\right)$ and (a) follows.

The following result follows now immediately from Proposition 2.6 and Lemma A.3.

Theorem A.4. Suppose that Assumption 2.3 holds. Let $u^{1}, u^{2} \in U$. Then, the set of all the $Q E$ of the game $\left(u^{1}(\phi), u^{2}(\phi)\right)$ induces finitely many quasi-distributions on outcomes iff for every $\omega \in \Omega$

$$
\operatorname{rank}\left(\begin{array}{cc}
u^{1}(\phi) & 0 \\
\phi^{\omega} & u^{2}(\phi) \\
0 & d_{n}
\end{array}\right)=2 k \quad \text { and } \quad \operatorname{rank}\left(\begin{array}{ccc}
u^{2}(\phi) & \phi^{\omega} & 0 \\
0 & u^{1}(\phi) & d_{m}
\end{array}\right)=2 k
$$

We address now the proof of Theorem 2.8. We show first the 'if' part. If the rank conditions in Theorem 2.8 hold, then by Theorem A.4 the set of QE induce a unique quasi-distribution on outcomes. Since the set of CMNE is a subset of the set of $\mathrm{QE}$, the set of CMNE also induces, at most, a unique distribution on outcomes. Thus, the 'if' part of Theorem 2.8 holds.

Conversely, suppose that the set of CMNE of the game $\left(u^{1}(\phi), u^{2}(\phi)\right)$ induces finitely many distributions on outcomes and that the game $\left(u^{1}(\phi), u^{2}(\phi)\right)$ has, at least, a CMNE, say $\bar{x}=x^{p}\left(u^{2}\right)+x^{h}\left(u^{2}, w_{0}\right) \in$ $\Delta_{+}\left(S^{1}\right)$ and $\bar{y}=y^{p}\left(u^{1}\right)+y^{h}\left(u^{1}, v_{0}\right) \in \Delta_{+}\left(S^{2}\right)$ with $w_{0} \in \mathbb{R}^{m-k}, v_{0} \in \mathbb{R}^{n-k}$. By continuity, there are open sets $H_{1} \subset \mathbb{R}^{n-k}$ and $H_{2} \subset \mathbb{R}^{m-k}$ such that for $v \in H_{1}, w \in H_{2}$ we have that $x=x^{p}\left(u^{2}\right)+x^{h}\left(u^{2}, w\right) \in$ $\Delta_{+}\left(S^{1}\right)$ and $y=y^{p}\left(u^{1}\right)+y^{h}\left(u^{1}, v\right) \in \Delta_{+}\left(S^{2}\right)$ is a CMNE of the game $\left(u^{1}(\phi), u^{2}(\phi)\right)$.

If the rank conditions in Theorem 2.8 do not hold, then, by Theorem A.4 for some outcome $\omega \in \Omega$ the polynomial $q_{\omega}(v, w)$ in $(7)$ is not constant and, hence, it takes a continuum of values as the variables $(v, w)$ vary on the open set $H_{1} \times H_{2}$. It follows that the set CMNE of the game $\left(u^{1}(\phi), u^{2}(\phi)\right)$ induce infinitely many distributions on outcomes, which contradicts our assumption. And the 'only if' part of Theorem 2.8 follows. 